\title{
Performance of Irradiated CVD Diamond Micro-Strip Sensors
}

\author{
RD42 Collaboration
}

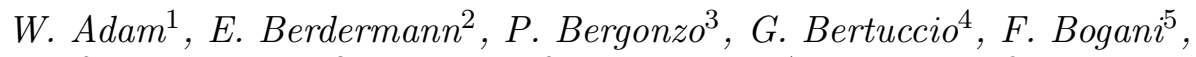
E. Borchi ${ }^{6}$, A. Brambilla ${ }^{3}$, M. Bruzzi ${ }^{6}, C$. Colledani $^{7}$, J. Conway ${ }^{8}$, P. D'Angelo, W. Dabrowski ${ }^{10}$, P. Delpierre ${ }^{11}$, A. Deneuville ${ }^{12}$,W. Dulinski ${ }^{7}$, B. van Eijk ${ }^{13}$, A. Fallou ${ }^{11}$, F. Fizzotti ${ }^{14}$, F. Foulon ${ }^{3}$, M. Friedl ${ }^{1}$, K.K. Gan ${ }^{15}$, E. Gheeraert ${ }^{12}$, G. Hallewell ${ }^{11}$, S. Han ${ }^{15}$, F. Hartjes ${ }^{13}$, J. Hrubec ${ }^{1}$, D. Husson ${ }^{7}$, H. Kagan ${ }^{15}$, D. Kania ${ }^{15}$, J. Kaplon ${ }^{16}$, R. Kass ${ }^{15}$, T. Koeth ${ }^{8}$, M. Krammer $^{1}$, A. Logiudice $^{14}$, R. Lu ${ }^{14}$, L. mac Lynne ${ }^{8}$, C. Manfredotti ${ }^{14}$, D. Meier ${ }^{16, \star}$, M. Mishina $^{18}$, L. Moroni ${ }^{9}$, J. Noomen ${ }^{13}$, A. Oh ${ }^{17}$, L.S. Pan ${ }^{15}$, M. Pernicka ${ }^{1}$, A. Peitz ${ }^{8}$, L. Perera ${ }^{8}$, S. Pirollo ${ }^{6}$, M. Procario ${ }^{19}$, J.L. Riester ${ }^{7}$, S. Roe ${ }^{16}$, L. Rousseau ${ }^{3}$, A. Rudge ${ }^{16}$, J. Russ ${ }^{19}$, S. Sala ${ }^{9}$, M. Sampietro ${ }^{4}$, S. Schnetzer ${ }^{8}$, S. Sciortino ${ }^{6}$, H. Stelzer ${ }^{2}$, R. Stone ${ }^{8}$, B. Suter ${ }^{19}$, R.J. Tapper ${ }^{20}$, R. Tesarek ${ }^{8}$,W. Trischuk ${ }^{21}$, D. Tromson ${ }^{3}$, E. Vittone ${ }^{14}$, A.M. Walsh ${ }^{8}, R$. Wedenig ${ }^{1}$, P. Weilhammer ${ }^{16}$, M. Wetstein ${ }^{8}$, C. White ${ }^{22}$,W. Zeuner ${ }^{17}$, M. Zoeller ${ }^{15}$

1 Institut für Hochenergiephysik der Österr. Akademie d. Wissenschaften, Vienna, Austria, 2 GSI, Darmstadt, Germany, ${ }^{3}$ LETI (CEA-Technologies Avancees) DEIN/SPE - CEA Saclay, Gif-Sur-Yvette, France, ${ }^{4}$ Polytechnico Milano, Italy, ${ }^{5}$ LENS, Florence, Italy, ${ }^{6}$ University of

Florence, Florence, Italy, ${ }^{7}$ LEPSI, IN2P3/CNRS-ULP, Strasbourg, France, ${ }^{8}$ Rutgers University, Piscataway, NJ, U.S.A., ${ }^{9}$ INFN, Milano, Italy, ${ }^{10}$ Faculty of Physics and Nuclear

Techniques, UMM, Cracow, Poland, ${ }^{11}$ CPPM, Marseille, France, ${ }^{12}$ LEPES, Grenoble,

France, ${ }^{13}$ NIKHEF, Amsterdam, Netherlands, ${ }^{14}$ Univerity of Torino, Italy, ${ }^{15}$ The Ohio State University, Columbus, OH, U.S.A., ${ }^{16}$ CERN, Geneva, Switzerland, ${ }^{17}$ II.Inst. für Exp.

Physik, Hamburg, Germany, ${ }^{18}$ FNAL, Batavia, U.S.A., ${ }^{19}$ Carnegie-Mellon University,

Pittsburgh, U.S.A., ${ }^{20}$ Bristol University, Bristol, U.K., ${ }^{21}$ University of Toronto, Toronto, ON, Canada, ${ }^{22}$ Illinois Institute of Technology, Chicago, IL, U.S.A.

\begin{abstract}
CVD diamond detectors are of interest for charged particle detection and tracking due to their high radiation tolerance. In this article we present, for the first time, beam test results from recently manufactured CVD diamond strip detectors and their behavior under low doses of electrons from a $\beta$-source and the performance before and after intense $\left(>10^{15} / \mathrm{cm}^{2}\right)$ proton- and pion-irradiations. We find that low dose irradiations increase the signal-to-noise ratio (pumping of the signal) and slightly deteriorate the spatial resolution. Intense irradiations with protons $(2.2 \times$ $\left.10^{15} \mathrm{p} / \mathrm{cm}^{2}\right)$ lowers the signal-to-noise ratio slightly. Intense irradiation with pions $\left(2.9 \times 10^{15} \pi / \mathrm{cm}^{2}\right)$ lowers the signal-to-noise ratio more. The spatial resolution of the diamond sensors improves after irradiations.
\end{abstract}

\footnotetext{
ऋ corresponding author: Dirk.Meier@cern.ch
} 


\section{Introduction}

The inherent properties of diamond indicate that it is a radiation resistant sensor material. Recent progress in the industrial production of chemical vapor deposition (CVD) diamond for detector applications and its fabrication into micro-strip and pixel devices may enable it to be used for radiation resistant particle tracking close to the interaction region at future experiments at high luminosity colliders $[1,2]$. The results presented in this article were obtained within the RD42 collaboration at CERN. The goal of the RD42 project is the development of CVD diamond detectors for the application at high luminosity experiments at the LHC and the Tevatron. This article presents the performance of recent CVD diamond micro-strip sensors in a particle beam and shows the performance of CVD diamond strip sensors after low dose electron illumination and after intense irradiation with protons and pions.

\section{Preparation of Diamond Strip Sensors}

The CVD diamond material was produced at De Beers [3] with a final thickness in the range from $490 \mu \mathrm{m}$ to $690 \mu \mathrm{m}$. Strips made of $\mathrm{Cr} / \mathrm{Au}$ were deposited on the growth side and a solid electrode on the nucleation side. The strip width was $25 \mu \mathrm{m}$ at a pitch of $50 \mu \mathrm{m}$. Charge sensitive amplifiers (VA2 VLSI readout chips from reference [4] with a signal peaking time of $2 \mu \mathrm{s}$ ) were directly wire bonded (dc-coupled) to the strips. A voltage was applied to the back electrode causing an electric field inside the diamond bulk. Fast charged particles (mips) that traverse the bulk deposit energy (the restricted energy loss of a mip is $145 \mathrm{keV}$ in $300 \mu \mathrm{m}$ diamond) and create charge carriers $(\approx 11000$ eh-pairs in $300 \mu \mathrm{m}$ diamond) that move along the electric field lines. The motion of these charge carriers induces an electric charge on the strips (induced current $\propto$ carrier velocity). The time integral of the induced current was measured by the charge sensitive amplifiers. The position of a particle was derived from the measured charge signal on the strips.

\section{Methods and Experimental Setup in the Beam}

Fig. 1 shows a schematic view of the silicon reference telescope [5] which was used in a $100 \mathrm{GeV} / c$ pion beam to study the performance of the diamond sensors before and after irradiations. The diamond sensors under study and its readout chips were mounted inside the telescope. The telescope allowed one

to measure the particle track and determine its intersection, at the position 
$\left(u_{\mathrm{t}}, v_{\mathrm{t}}\right)$, in the diamond plane with a precision of about $1 \mu \mathrm{m}[6]$. All detector planes were parallel to each other and only events with single tracks were used.

Silicon Strip

Reference Detectors

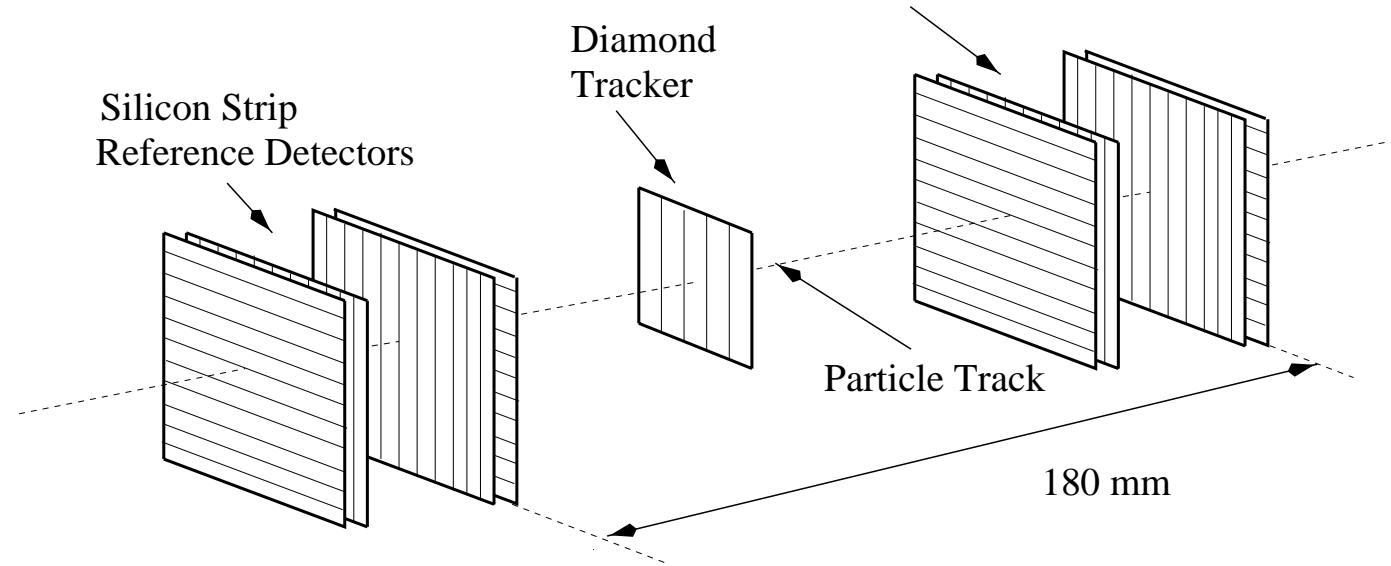

Fig. 1. Schematic view of the silicon beam telescope used for characterization of diamond strip sensors.

The 2-strip transparent analysis method was used to measure the sum of charge signals, $Q=q_{1}+q_{\mathrm{r}}$, on the strips closest to the left and to the right of the particle track. It was shown that for the $50 \mu \mathrm{m}$ pitch most charges are collected by two strips, left and right of the intersection point [7]. In the following the distributions of 2-strip transparent signals will be shown in units of the equivalence noise charge, $\sigma_{\mathrm{ENC}}$, on one strip. The equivalent noise charge was measured using pedestal subtracted and common mode corrected raw values from strips far away $(>1 \mathrm{~mm})$ from the particle intersection. For the sensors measured here the equivalent noise charge corresponds to $\sigma_{\mathrm{ENC}}=(110 \pm 10) e$. The ratio signal-to-noise, $s=Q / \sigma_{\mathrm{ENC}}$, is dimensionless and independent of the electronic gain of the readout and acquisition system. In $N$ successive events a signal-to-noise distribution, $\mathrm{d} N(s) / \mathrm{d} s$, was acquired which allows one to derive its mean value, $\bar{s}$, its most probable value, $\hat{s}$, and the full width at half the height, $s_{\text {FWHM }}$, before and after irradiation. These values are used in the following to quantify the electronic quality of the diamond sensors before and after irradiation.

The spatial resolution of the detector quantifies the precision with which the position of the intersection can be measured by the diamond detector. The charge signals from two strips, those with the largest charge signal, $q_{0}$ at position $u_{0}$, and the next adjacent strip with the next highest charge, $q_{1}$ at position $u_{1}$, were used to measure the position, $u_{\mathrm{h}}$, of the intersection in the direction perpendicular to the strips. Using the 2-strip center-of-gravity method the position is given by $u_{\mathrm{h}}=\left(q_{0} u_{0}+q_{1} u_{1}\right) /\left(q_{0}+q_{1}\right)$. It was shown previously that the two strip center-of-gravity method gives a better precision on the measurement than the single strip method or the 3-strip center-of-gravity method [7]. Using the measurement, $u_{\mathrm{t}}$, from the beam reference telescope one derives 
the residual, $r=u_{\mathrm{h}}-u_{\mathrm{t}}$. Residual distributions, $\mathrm{d} N(r) / \mathrm{d} r$, were fitted by Gaussian functions with standard deviations, $\sigma$. The standard deviation of the Gaussian fit is the spatial resolution of the diamond strip sensor.

\section{Irradiation with Electrons from a $\beta$-Source}

It is known that the charge signal from diamond samples with large circular contacts increases under illumination with charged particles ('pumping effect') $[8,9]$. On unirradiated samples an increase by a factor of 1.6 to 2.0 is observed for an absorbed dose below 10 Gy. Above 10 Gy the charge signal remains constant.

Here we report the observation of 'pumping' in diamond strip sensors. Fig.2 shows the 2-strip transparent signal-to-noise distributions before and after illumination with electrons from a ${ }^{90} \mathrm{Sr} \beta$-source (pumped). Both distributions are Landau-like and their mean, most probable values and width are shown. It can be seen that after illumination the signal-to-noise values are increased. The mean value increased from 32 to 59 by a factor of 1.8 which is within the range expected for diamond samples. The noise was measured separately before and after illumination and found to remain constant. Hence one infers an increase of the signals on strips under illumination with electrons. We note that the pumped state remains stable in time as long a the sensor is kept in darkness and at room temperature. An absorbed dose of about (1 to 10) Gy was estimated from the activity of the source $(37 \mathrm{MBq})$, the time of illumination (a few hours) and the distance between source and sensor during illumination $(4 \mathrm{~cm})$. Fig. 3 shows the residual distributions measured from the same diamond sensor before and after the same electron illumination. It can be seen that the residual distribution is narrower before electron illumination and the spatial resolution slightly deteriorates from $(10.8 \pm 0.3) \mu \mathrm{m}$ to $(12.4 \pm 0.4) \mu \mathrm{m}$. Table 4 summarizes the results from this diamond strip sensor and a second one tested in the beam before and after electron illumination. Both sensors behave similarly with respect to pumping and spatial resolution. Hence one may consider low dose illumination with electrons (pumping) to be beneficial for the signal-to-noise and for the efficiency of diamond strip sen-

sors while it occurs less beneficial for the spatial resolution. In the following the data on heavily proton and pion irradiated diamond sensors are shown in the (electron) pumped state only. We note that diamond trackers in a high luminosity experiment will natually operate in the pumped state due to the large amount of charged particles. 


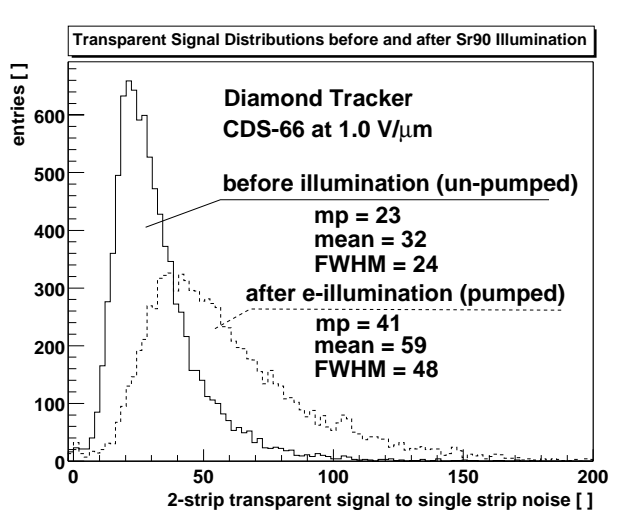

Fig. 2. Distributions of 2-strip transparent signal-to-noise before (solid line) and after (dashed line) electron illumination.

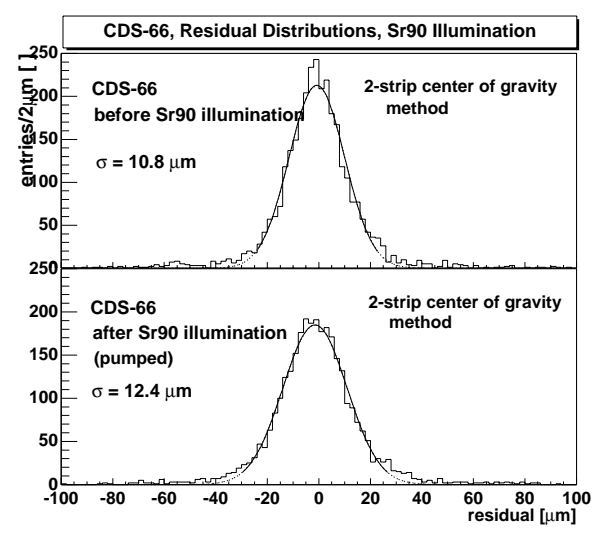

Fig. 3. Distributions of the residuals before (upper) and after (lower) electron illumination.

\begin{tabular}{|c|c|c|c|c|c|}
\hline $\begin{array}{c}\text { CVD diamond } \\
\text { sensor }\end{array}$ & $\begin{array}{c}\text { thickness } \\
{[\mu \mathrm{m}]}\end{array}$ & state & $\begin{array}{l}2 \text {-str } \\
\text { mean } \\
\bar{s}\end{array}$ & $\begin{array}{c}\text { p transparent } \\
\text { most probable } \\
\hat{s}\end{array}$ & $\begin{array}{c}\text { 2-strip c.o.g. } \\
\text { spatial resolution } \\
{[\mu \mathrm{m}]}\end{array}$ \\
\hline \multirow{2}{*}{ CDS-65 } & \multirow{2}{*}{520} & as-made & 33 & 24 & 11.3 \\
\hline & & pumped & 65 & 44 & 13.3 \\
\hline \multirow{2}{*}{ CDS-66 } & \multirow{2}{*}{525} & as-made & 32 & 23 & 10.8 \\
\hline & & pumped & 59 & 41 & 12.4 \\
\hline
\end{tabular}

Table 1

Performance of two CVD diamond sensors in the beam before (as-made) and after illumination with electrons from the $\beta$-source (pumped).

\section{Performance after Proton Irradiation}

While results on proton irradiated diamond samples with large circular contacts were reported previously we are now studying the performance of proton irradiated diamond strip sensors. The irradiations were performed using $24 \mathrm{GeV} / c$ protons from the Proton Synchrotron (PS) at CERN where details of the irradiation can be found in reference [10]. The absorbed dose can be derived from the proton fluence. For the sensors irradiated here one finds that $1 \times 10^{15} \mathrm{p} / \mathrm{cm}^{2}$ corresponds to about 0.23 MGy in diamond.

Fig. 4 shows the transparent 2-strip signal-to-noise distributions measured on a diamond sensor in beam tests before and after $1 \times 10^{15} \mathrm{p} / \mathrm{cm}^{2}$ and $2.2 \times$ $10^{15} \mathrm{p} / \mathrm{cm}^{2}$. While the strip contacts before and after $1 \times 10^{15} \mathrm{p} / \mathrm{cm}^{2}$ were still the same we note that the contacts were replaced after $2.2 \times 10^{15} \mathrm{p} / \mathrm{cm}^{2}$ and then characterized in the test beam. We also note that the beam tests were 
carried out with the diamond being in its pumped state. At $1 \times 10^{15} \mathrm{p} / \mathrm{cm}^{2}$ we observe that the shape of the signal-to-noise distribution is narrower than before irradiation and entries in the tail of the distribution appear closer to the most probable signal. At $2.2 \times 10^{15} \mathrm{p} / \mathrm{cm}^{2}$ and after re-metalization we observe essentially the same signal-to-noise distribution like at $1 \times 10^{15} \mathrm{p} / \mathrm{cm}^{2}$ indicating that no further damage occured to the diamond itself. The most probable signal-to-noise was 41 before irradiation and 35 at $1 \times 10^{15} \mathrm{p} / \mathrm{cm}^{2}$ and also at $2.2 \times 10^{15} \mathrm{p} / \mathrm{cm}^{2}$. Therefore we find a reduction of maximum $15 \%$ in the most probable signal-to-noise after irradiation with $2.2 \times 10^{15} \mathrm{p} / \mathrm{cm}^{2}$. The noise was measured to remain constant at each beam test. Since the beam test at $2.2 \times 10^{15} \mathrm{p} / \mathrm{cm}^{2}$ used new contacts the observed decrease of $15 \%$ is due to damage in the diamond itself.

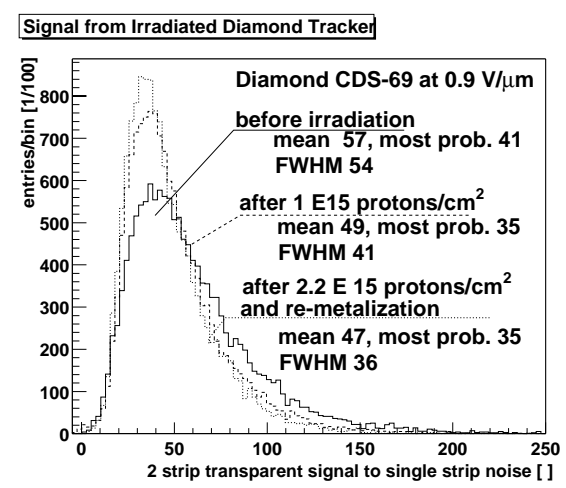

Fig. 4. Transparent 2-strip charge signal-to-noise distributions before (solid line), after proton irradiations with $1 \times 10^{15} \mathrm{p} / \mathrm{cm}^{2}$ (dashed line) and after $2.2 \times 10^{15} \mathrm{p} / \mathrm{cm}^{2}$ (dotted line).

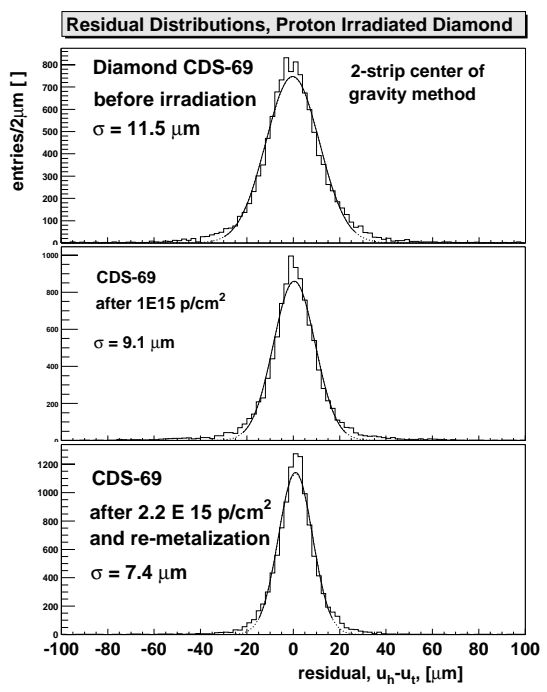

Fig. 5. Residual distributions measure before and after proton irradiations.

Fig. 5 shows residual distributions before and after irradiation at $1 \times 10^{15} \mathrm{p} / \mathrm{cm}^{2}$ and $2.2 \times 10^{15} \mathrm{p} / \mathrm{cm}^{2}$. We observe that the spatial resolution improves from $11.5 \pm 0.3) \mu \mathrm{m}$ before irradiation over $(9.1 \pm 0.3) \mu \mathrm{m}$ at $1 \times 10^{15} \mathrm{p} / \mathrm{cm}^{2}$ to $(7.4 \pm 0.2) \mu \mathrm{m}$ at $2.2 \times 10^{15} \mathrm{p} / \mathrm{cm}^{2}$. A spatial resolution of nearly $7 \mu \mathrm{m}$ is a very good result for any sensor with $50 \mu \mathrm{m}$ strip pitch without intermediate strips.

\section{Performance after Pion Irradiation}

While experimental methods and results of pion irradiated diamond samples with large circular contacts can be found in references $[11,12,1,13]$ we are reporting here results from the first pion irradiated diamond strip sensor. The 
irradiations were performed using $300 \mathrm{MeV} / c$ pions $\left(\pi^{+}\right)$available at the Paul Scherrer Institute in Switzerland. The absorbed dose was derived from the pion fluence. For the sensors irradiated here one finds that $1 \times 10^{15} \pi / \mathrm{cm}^{2}$ corresponds to about $0.23 \mathrm{MGy}$ in diamond. The energy deposited by $300 \mathrm{MeV} / \mathrm{c}$ is close to a mip and hence assumed to be comparable to the energy deposited by $24 \mathrm{GeV} / c$ protons.

After irradiation we observed that most of the strips were damaged: the metal $(\mathrm{Cr} / \mathrm{Au})$ appeared to be detached from the diamond surface such that wire bonding would have been impossible. Similar observations were recently made on diamond samples with circular contacts [13]. The reason for the contact damage is under study. Subsequently the diamond sensor was re-metalized and characterized in the test beam. Fig. 6 shows the transparent 2-strip signal-tonoise distribution measured on the diamond sensor in a beam test before and after irradiation with $2.9 \times 10^{15}$ pions $/ \mathrm{cm}^{2}$. The measurements in the beam tests were carried out with the diamond being in its pumped state. We observe that the shape of the distribution is narrower than before irradiation and entries in the tail of the distribution appear closer to the most probable signal. The most probable signal-to-noise was at 44 before irradiation and at 21 after irradiation. Therefore we find a reduction by $50 \%$ in the most probable signal-to-noise after irradiation with $2.9 \times 10^{15} \mathrm{p} / \mathrm{cm}^{2}$. The noise was measured to remain constant. Since the contacts were replaced we may attribute the reduction to radiation damage in the diamond itself.
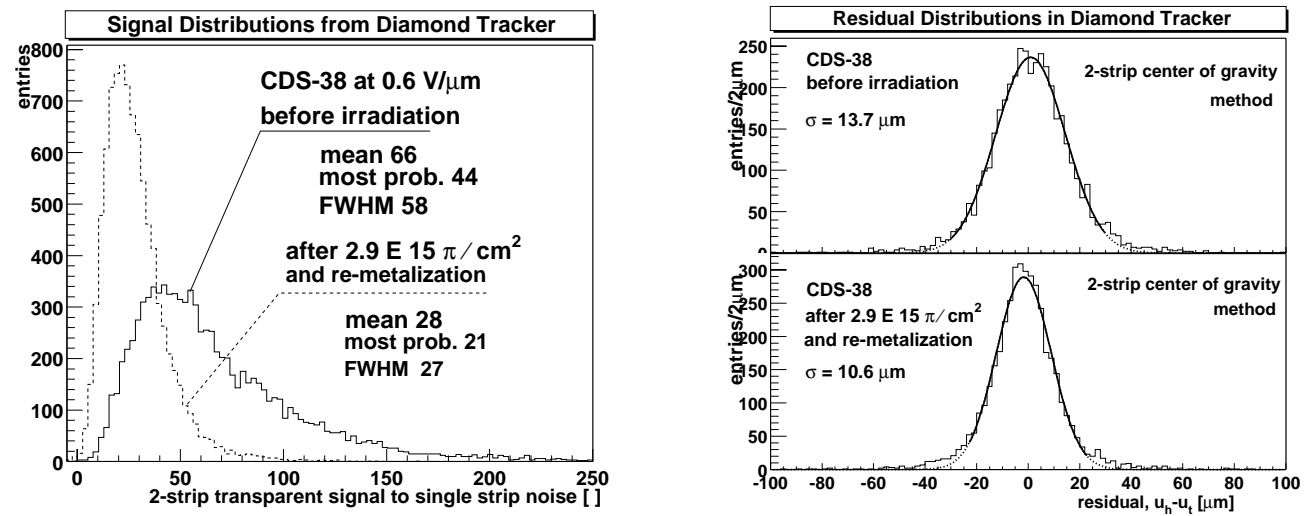

Fig. 6. Transparent 2-strip charge signal-to-noise distributions before (solid line), after pion irradiation with $2.9 \times 10^{15} \pi / \mathrm{cm}^{2}$ (dashed line).

Fig. 7. Residual distributions before and and after pion irradiation.

Fig. 7 shows residual distributions before and after irradiation with $2.9 \times$ $10^{15} \mathrm{p} / \mathrm{cm}^{2}$. We observe that the spatial resolution improves from $(13.7 \pm$ $0.4) \mu \mathrm{m}$ before irradiation to $(10.6 \pm 0.3) \mu \mathrm{m}$. Together with the observation on proton irradiated diamond we infer that intense irradiation in general improves spatial resolution in CVD diamond sensors. 


\section{Summary and Discussion}

Results from beam tests on irradiated CVD diamond micro-strip sensors have been presented. A beneficial increase of the signal-to-noise (pumping effect) by a factor 1.6 to 2 was observed after low dose (below $10 \mathrm{~Gy}$ ) illumination with electrons from a ${ }^{90} \mathrm{Sr} \beta$-source. Although the leakage current typically increases on pumped samples it is too low to cause an increase in channel readout noise. Indeed no significant increase in noise was measured after pumping indicating a true increase of the signal. The leakage current in the beam is typically below $5 \mathrm{nA} / \mathrm{cm}^{2}$ at $1 \mathrm{~V} / \mu \mathrm{m}$ which is very low and contributes with less than $30 e$ to the total readout noise. The spatial resolution deteriorates slightly (10\% to $20 \%$ ) but remains clearly below digital resolution of $50 \mu \mathrm{m} / \sqrt{12} \approx 14.4 \mu \mathrm{m}$. Irradiations with protons indicate a reduction of maximum $15 \%$ in the most probable signal-to-noise after $2.2 \times 10^{15} \mathrm{p} / \mathrm{cm}^{2}$ while the noise was measured to remain constant. Irradiations with pions resulted in visible damage to the $(\mathrm{Cr} / \mathrm{Au})$ contacts and required re-metalization. Still after re-metalization only $50 \%$ of the signal was observed on the tracker irradiated with $2.9 \times 10^{15} \pi / \mathrm{cm}^{2}$. It is not understood why pions cause contact damage and eventually larger damage in diamond than protons. However pions have similar beneficial effect on spatial resolution like protons. The improvements in spatial resolution after irradiations is assumed to go along with an improved signal uniformity after irradiations.

In the future radiation effects in diamond sensors should be considered to be related to the diamond material on one hand and contact damage on the other. However it is difficult to separate both effects due to problems in characterizing the contacts. In the future contacts will be prepared using $\mathrm{Ti} / \mathrm{Au}$ which may be more radiation resistant. Finally we note that the fluences of $2.2 \times 10^{15} \mathrm{p} / \mathrm{cm}^{2}$ and $2.9 \times 10^{15} \pi / \mathrm{cm}^{2}$ are very high (corresponding to more than $500 \mathrm{kGy}$ energy deposited in diamond) and exceed those expected for high luminosity experiments. The performance of the diamond sensors after these high fluences here is still very good.

\section{Acknowledgements}

We would like to thank M. Glaser at CERN for their work on proton irradiations. We also thank the organizers of the "Conference on Radiation Effects on Semi-Conductor Materials, Detectors and Devices" for this very pleasant and interesting meeting. 


\section{References}

[1] W. Adam et al. (RD42-Collaboration). "Development of Diamond Tracking Detectors for High Luminosity Experiments at the LHC". Status Report/RD42, CERN/LHCC 2000-011, (2000).

[2] S. Schnetzer et al. (RD42 Collaboration). "Tracking with CVD Diamond Radiation Sensors at High Luminosity Colliders". IEEE Trans. Nucl. Sci., 46 (1999) 193-200.

[3] De Beers Industrial Diamond Division (UK) Ltd. Charters, Sunninghill, Ascot, Berkshire, SL5 9PX England.

[4] Integrated Detector and Electronics (IDE) AS. "The VA Circuits". www.ideas.no. Pb.315, Veritasveien 9, N-1322 Hovik, Norway.

[5] C. Colledani et al. "A Submicron Precision Silicon Telescope for Beam Test Purposes". Nucl. Instr. Meth., A372 (1997) 3.

[6] D. Meier. "CVD Diamond Sensors for Particle Detection and Tracking". $\mathrm{PhD}$ thesis, University of Heidelberg and CERN, atlasinfo.cern.ch/Atlas/ documentation/thesis/thesis.html, (1999).

[7] W. Adam et al. (RD42-Collaboration). "Micro-Strip Sensors Based on CVD Diamond". In INSTR99, Hamamatsu, Japan, Preprint at CERN-EP-2000-041.

[8] S. Zhao. "Characterization of the Electrical Properties of Polycrystalline Diamond Films". PhD thesis, Ohio State University, (1994).

[9] W. Adam et al. (RD42-Collaboration). "Development of CVD Diamond Radiation Detectors". Proc. $5^{\text {th }}$ Symp. Diamond Mat., Electr. Chem. Soc., Paris, Preprint CERN-EP/98-80, (1997).

[10] D. Meier et al. (RD42-Collaboration). "Proton Irradiation of CVD Diamond Detectors for High Luminosity Experiments at the LHC". Nucl. Instr. Meth., A426 (1999) 173.

[11] C. Bauer et al. (RD42-Collaboration). "Pion Irradiation Studies of CVD Diamond Detectors". Preprint CERN-PPE/95-173, (1995).

[12] W. Adam et al. (RD42-Collaboration). "Development of Diamond Tracking Detectors for High Luminosity Experiments at the LHC". Status Report/RD42, CERN, (June 1998). LHCC 98-20, www. cern.ch/RD42.

[13] W. Adam et al. (RD42 Collaboration). "Radiation Tolerance of CVD Diamond Detectors for Pions and Protons". In Proc. $3^{\text {rd }}$ Int. Conf. Radiation Effects on Semi-conductor Materials, Detectors and Devices. Florence, Italy, (2000). 